\title{
Influence of ezrin-shRNA in combination with HSP70 on the apoptosis and proliferation of osteosarcoma cells
}

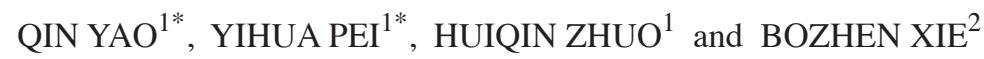 \\ ${ }^{1}$ Central Laboratory; ${ }^{2}$ Department of Spine Surgery, Zhongshan Hospital Xiamen University, \\ Xiamen, Fujian 361004, P.R. China
}

Received April 9, 2015; Accepted July 26, 2016

DOI: $10.3892 / \mathrm{ol} .2016 .5103$

\begin{abstract}
Ezrin and heat shock protein (HSP)70 have been reported to regulate cell apoptosis and tumor development of osteosarcoma. However, there has not been reported the synergy effect of knocking down ezrin and overexpressing HSP70. In the present study, two vectors, pGFP-V-RS-shRNA and pGFP-V-RS-shRNA-HSP70, were constructed and transfected into LM8 cells [denoted as small hairpin (sh)RNA group and dual group, respectively]. The apoptosis rates in these two transfected groups were significantly higher than those in the control group (empty vector) $(\mathrm{P}=0.036)$, while significantly lower proliferation rates were observed in these two groups $(\mathrm{P}=0.023)$. The cytotoxic T lymphocyte activity on target LM8 tumor cells in the dual group was significantly higher than in other groups, with cytotoxicity as high as $55.56 \pm 2.10 \%$. Further studies revealed that the transfection of ezrin-shRNA/HSP70 also suppressed tumor formation in vivo in nude mice. A lower cluster of differentiation (CD)4/CD8 ratio was detected in the tumor formed by injecting cells in the dual group $(\mathrm{P}=0.006)$. Furthermore, the serum level of interleukin- 4 in the dual group was significantly decreased, while the serum level of interferon- $\gamma$ was significantly increased, compared with the other two groups $(\mathrm{P}=0.004)$. Simultaneously knocking down ezrin and overexpressing HSP70 promotes cellular apoptosis and suppresses the proliferation of osteosarcoma cells in vitro, and enhances the tumor killing effects of HSP70-induced immune killing.
\end{abstract}

\section{Introduction}

Osteosarcoma is the most common primary malignant bone tumor, and usually occurs in adolescents and children (peak

Correspondence to: Dr Bozhen Xie, Department of Spine Surgery, Zhongshan Hospital Xiamen University, 201 Hubin South Road, Xiamen, Fujian 361004, P.R. China

E-mail: xiezhenbo88@126.com

*Contributed equally

Key words: shRNA, ezrin, HSP70, apoptosis, immune killing of incidence, $\sim 18$ years old) (1). The cancer causes tremendous disfiguration as a result of amputation, and has a high morbidity and mortality rate (2). For these reasons, it is of great importance to clarify the mechanisms that underlie the cause, occurrence and development of osteosarcoma in order to identify more effective approaches for its treatment. Systemic chemotherapy following surgical removal of the tumor has been an effective therapeutic method for the treatment of osteosarcoma (3). However, major problems, including cytotoxic side effects and drug resistance, are associated with chemotherapy $(4,5)$. Thus, safe and more effective anti-cancer treatments are required for patients with osteosarcoma.

Ezrin is currently considered one of the reasonable and effective targets for cancer gene therapy (6). Ezrin, a membrane cytoskeletal cross-linker, belongs to the ezrin/radixin/moesin protein family, and is involved in the regulation of the cell cycle, cell proliferation, cell differentiation and apoptosis (6,7). Ezrin protein expression was reported to be significantly increased in osteosarcoma tumors, and its levels are negatively correlated with patient 5 -year survival rates (8-10). As a potential effective target, the silencing or downregulation of ezrin expression may be an effective approach to suppress tumor cell proliferation and to improve patient survival rate $(11,12)$.

It is difficult to completely knockout or inhibit the expression of a single gene to kill tumor cells by current transgenic technologies, which explains why incomplete tumor removal, recurrence and metastasis remain a challenge in tumor treatment (13). Therefore, extensive efforts have been paid to the selection of a combination of target genes to achieve a better curative effect $(14,15)$. Heat shock protein (HSP) 70 , which is an adenosine triphosphate-dependent molecular chaperone, regulates protein conformation, stability and interactions (16). The majority of HSP70 ligands are proteins essential for cell survival and growth, including protein kinases, steroid receptors and transcription factors $(16,17)$. In addition, when tissue damage or tumorigenesis occurs, HSPs are abundantly expressed, and form complexes with peptides (18). If the peptides are generated in normal tissues, the HSP70-peptide complexes do not induce an immune response; however, if the peptides are tumor-derived mutated antigens, the HSP70-peptide complexes can be presented effectively to immune cells, thus breaking the immune tolerance and inducing the tumor killing effects of T lymphocytes and natural killer (NK) cells $(18,19)$. 
Therefore, it was hypothesized that inhibiting proliferation and promoting apoptosis of tumorcells by simultaneously providing exogenous danger signals such as overexpressing HSPs would promote the recognition and presentation of tumor antigenic peptides by antigen-presenting cells (APCs) (20), enhance the immunogenicity of tumor-associated antigens (21) and tumor-specific antigens (22) derived from membrane molecules of apoptotic tumor cells, and induce the active anti-tumor immune response mediated by specific $\mathrm{T}$ lymphocytes, thus enhancing their killing effects on tumor cells and removing the remaining tumor cells in patients. Recently, the present authors observed that simultaneously knocking down ezrin and overexpressing HSP70 promoted the apoptosis and inhibited the proliferation of human osteosarcoma cells (23). Based on the unique features of ezrin and HSP70, a specific vector was designed and constructed in the present study to simultaneously knock down ezrin expression and upregulate HSP70 expression. Stably transfected LM8 osteosarcoma cell lines with this vector were established and used to analyze the influence of ezrin-small hairpin (sh)RNA in combination with HSP70 on cell growth, proliferation, apoptosis and HSP70-induced cytotoxic T lymphocyte (CTL) activity in vitro. In addition, the suppression of proliferation and the tumor killing effects on LM8 cells were assessed. Furthermore, tumor-bearing mice were prepared by injection with the stably transfected cells, and the inhibitory effects of ezrin knock-down in combination with HSP70-induced immune response on tumor growth in vivo were analyzed. The results obtained in the present study provide the basis for a novel method of gene therapy for osteosarcoma based on suppressing the proliferation and promoting the apoptosis of tumor cells, in addition to inducing dual effects of specific immune response.

\section{Materials and methods}

Cell culture. The murine osteosarcoma cell line LM8 was purchased from the China Center for Type Culture Collection (Wuhan, China), and was cultured in Dulbecco's modified Eagle medium (DMEM; HyClone; GE Healthcare Life Sciences, Logan, UT, USA) supplemented with $10 \%$ fetal bovine serum (HyClone; GE Healthcare Life Sciences) at $37^{\circ} \mathrm{C}$ in a $5 \% \mathrm{CO}_{2}$ incubator.

Vector construction and transient transfection. Ezrin-shRNA containing a hairpin loop was designed according to the ezrin messenger(m)RNAcomplementary(c)DNAsequence(GenBank, BC013903.2; http://www.ncbi.nlm.nih.gov/gene/22350). The sequences of the primers used were 5'-TGTATGAGCCTG TGAATT-TTCAAGAGA-AATTCACAGGCTCATACATT-3' and 5'-TGGAGGCCAAAGTACCACAC-3', in which there was a recognition site for $\mathrm{BamHI}$ at the $5^{\prime}$ end and a recognition site for HindIII at the $3^{\prime}$ end. The sequences were synthesized and cloned into the pGFP-V-RS vector (OriGene Technologies, Inc., Rockville, MD, USA) to generate the pGFP-V-RS-shRNA vector. Then, the vector was transformed into JM10 cells (obtained from China Center for Type Culture Collection, Wuhan, China), which were amplified and selected by puromycin resistance. Sequence identification of the ezrin gene cloned in the vector was performed by Invitrogen (Thermo Fisher Scientific, Inc., Waltham, MA, USA).

The HSP70 DNA sequence was synthesized according to its mRNA sequence (GenBank, NM_010478.2). Then, the green fluorescent protein (GFP) coding sequence in the pGFP-V-RS vector was substituted by the GFP-HSP70 coding sequence by enzymatic digestion and ligation in order to generate the pGFP-V-RS-HSP70 vector, which was transformed into DH5 $\alpha$ cells (Gene Company Ltd., Shanghai, China), amplified and selected. Sequence identification of the HSP70 gene cloned in the vector was performed by Invitrogen (Thermo Fisher Scientific, Inc.). A similar method was used to construct the pGFP-V-RS-shRNA-HSP70 vector.

Vectors, including empty vector pGFP-V-RS (control group), pGFP-V-RS-shRNA (shRNA group) and pGFP-V-RS-shRNA-HSP70 (dual group), were transfected into LM8 cells using Lipofectamine 2000 (Invitrogen; Thermo Fisher Scientific, Inc.) according to the manufacturer's protocol. The average transfection efficiency was 50-70\%. Cells were allowed to recover in medium for $24 \mathrm{~h}$ after transfection. All experiments were performed in 6-well tissue culture plates with cells plated to reach $50-60 \%$ confluence on the day of transfection.

Reverse transcription-quantitative polymerase chain reaction (RT-qPCR). Total RNA from cultured cells was isolated using TRIzol reagent (Invitrogen; Thermo Fisher Scientific, Inc.). The 260/280 absorbance ratio was measured for verification of the purity of RNA. RNA samples were reverse transcribed into cDNA using M-MLV Reverse Transcriptase (Promega Corporation, Madison, WI, USA). The sequences of the ezrin, HSP70 and 18S ribosomal (r)RNA genes were obtained from the GenBank database, and specific primers for them were designed by Primer Premier 5.0 software (Premier Biosoft International, Palo Alto, CA, USA). The following human primers were used: Ezrin, forward 5'-ACT CACCAGAAACCGAAAATG-3' and reverse 5'-TGGAGG CCAAAGTACCACAC-3'; HSP70, forward 5'-AAGAGCAAC AGCAGCAGACA-3' and reverse 5'-CGATTGGCAGGTCCA CAGTA-3'; and 18S rRNA, forward 5'-CCTGGATACCGC AGCTAGGA-3' and reverse 5'-GCGGCGCAATACGAA TGCCCC-3'. RT-qPCR was performed using SYBR Green qPCR SuperMix (Invitrogen; Thermo Fisher Scientific, Inc.) according to the manufacturer's protocol, and glyceraldehyde 3-phosphate dehydrogenase (GAPDH) served as an internal control. The thermal cycling conditions were as follows: $95^{\circ} \mathrm{C}$ for $3 \mathrm{~min}, 25$ cycles of $95^{\circ} \mathrm{C}$ for $30 \mathrm{sec}, 55^{\circ} \mathrm{C}$ for $2 \mathrm{~min}$ and $72^{\circ} \mathrm{C}$ for $30 \mathrm{sec}$, and a final step of $72^{\circ} \mathrm{C}$ for $5 \mathrm{~min}$. qPCRs for all samples were repeated three times.

Western blot analysis. The cells were washed twice in ice-cold phosphate-buffered saline (PBS) and resuspended in $5 \mathrm{v} / \mathrm{v}$ of ice-cold lysis buffer [20 mM 4-(2-hydroxyethyl)-1-piperazineethanesulfonic acid- $\mathrm{KOH}, 1.5 \mathrm{mM} \mathrm{MgCl} \mathrm{Mg}_{2}, 1 \mathrm{mM}$ ethylenediaminetetraacetic acid, $1 \mathrm{mM}$ ethylene glycol-bis( $\beta$-aminoethyl ether)-N,N,N',N'-tetraacetic acid, $1 \mathrm{mM}$ dithiothreitol and $0.1 \mathrm{mM}$ phenylmethanesulfonyl fluoride ( $\mathrm{pH}$ 7.5)]. The resuspended cells were homogenized with 10 pulses in a Teflon ${ }^{\circledR}$ homogenizer to extract the total protein. Protein samples $(10 \mu \mathrm{g})$ were separated by sodium dodecyl sulfate-polyacrylamide gel electrophoresis in 12 and 
$8 \%$ polyacrylamide gels. The separated proteins were then electrotransferred to a polyvinylidene fluoride membrane. Upon blocking in 5\% non-fat milk for $1 \mathrm{~h}$, the membrane was incubated at room temperature for $1 \mathrm{~h}$ with primary antibodies against ezrin (1:1,000; \#3145), HSP70 (1:1,000; \#4872) (CST Biological Reagents Company Limited, Shanghai, China), GAPDH (1:2,000; \#TA309157; Shanghai KangChen Bio-tech Inc., Shanghai, China), B-cell lymphoma (Bcl)-2 (1:1,000; \#2872), Bcl-2 associated X protein (Bax; 1:1,000; \#2772) and cyclin D1 (1:1,000; \#2922) (CST Biological Reagents Company Limited). The membrane was washed with Tris-buffered saline and Tween 20 (TBST) three times for 5 min each, and incubated at room temperature for $2 \mathrm{~h}$ in TBST containing horseradish peroxidase (HRP)-conjugated goat anti-rabbit immunoglobulin G antibody (1:20,000; SouthernBiotech, Birmingham, AL, USA). The membrane was next washed with TBST three times for $10 \mathrm{~min}$ each, and then incubated for $30 \mathrm{sec}$ with Pierce ECL Western Blotting Substrate (Thermo Fisher Scientific, Inc.) reagent for the development of the HRP signals, followed by exposure to autoradiography film for visualization of the bands. GAPDH was used as a loading control.

Analysis of apoptosis. Cellular apoptosis was determined using an annexin V-fluorescein isothiocyanate (FITC) apoptosis detection kit (Clontech Laboratories Inc., Mountainview, CA, USA). Brieflly, cells were cultured at a density of $4 \times 10^{6}$ cells $/ \mathrm{ml}$ and seeded in 6 -well plates. Cells were harvested by trypsinization, washed twice with cold PBS and centrifuged at $100 \times \mathrm{g}$. Cells $\left(1 \times 10^{5}-1 \times 10^{6}\right)$ were then resuspended in $300 \mu 1 \mathrm{X}$ binding buffer and centrifuged again at $100 \mathrm{x} g$ for $5 \mathrm{~min}$. The supernatant was next removed, and $10 \mu \mathrm{l}$ annexin V-FITC was added to the cells, which were incubated in the dark for $30 \mathrm{~min}$ at room temperature. Subsequently, cells were incubated in the dark with $5 \mu \mathrm{l}$ propidium iodide, and analyzed by flow cytometry (BD FACSCalibur ${ }^{\mathrm{TM}}$; BD Biosciences, Franklin Lakes, NJ, USA). The test for each sample was repeated three times, and data were represented as the mean value.

Analysis of cell proliferation. Cell proliferation was determined by 3-(4,5-dimethylthiazol-2-yl)-5-(3-carboxymethoxy phenyl)-2-(4-sulfophenyl)-2H-tetrazolium (MTT) assay. The transfected cells were plated in 96-well plates at a density of $0.1-0.2 \times 10^{4}$ cells/well. Next, $20 \mu \mathrm{l} \mathrm{MTS} /$ phenazine methosulfate mixture was added to each well, and cells were incubated for 3-4 h. The absorbance was then determined at an optical density (OD) of $570 \mathrm{~nm}$. The cell proliferation was measured over 7 days. The experiment was repeated three times, and data were represented as the mean absorbance value. The cell growth curve was represented to compare the growth rates upon transfection, and the proliferation rates and proliferation inhibitory rates were calculated.

Preparation of mouse spleen lymphocytes. BALB/c mouse (Yunnan Animal Center, Kunming, China) spleens were removed under non-sterile conditions $\left(25^{\circ} \mathrm{C}\right.$, cycle of day/night of $12 \mathrm{~h} / 12 \mathrm{~h}$, fed twice per day), cut into small pieces with sterile scissors and pushed through a stainless steel screen (100-mesh) in Hank's solution (pH 7.2-7.6). The spleen cell suspension was prepared and used to isolate mononuclear cells (lymphocytes and monocytes) with a lymphocyte isolation solution (Sigma-Aldrich; Merck Millipore, Darmstadt, Germany). The isolated cells were suspended at a concentration of $1 \times 10^{6}$ cells/ml in RPMI 1640 supplemented with $15 \%$ newborn calf serum (Thermo Fisher Scientific, Inc.). Spleen lymphocytes $\left(1 \times 10^{6}\right)$ were resuspended in the culture supernatant of LM8 cells that had been stably transfected with pGFP-V-RS-shRNA-HSP70. Recombinant interleukin-2 (rIL-2) was added to the mixture at 2,000 U/ml. After culture at $37^{\circ} \mathrm{C}$ for 7 days, the cells were harvested and used as sensitized mouse spleen CTL. Control spleen lymphocytes were isolated as described above and treated with rIL-2 (2,000 U/ml) but without the culture supernatant.

CTL killing assays. LM8 osteosarcoma cells in logarithmic growth phase were harvested and suspended at a concentration of $2 \times 10^{6}$ cells $/ \mathrm{ml}$ in RPMI 1640 medium, serving as target (T) cells. Upon amplification, the induced specific CTLs, including the sensitized and non-sensitized spleen lymphocytes, served as effector (E) cells. The E and T cells were mixed at 100:1, 50:1 and 25:1 (E:T) ratios, and seeded in 96-well plates (200 $\mu \mathrm{l} /$ well). For controls, $100 \mu \mathrm{l} /$ well E lymphocytes or T cells were seeded, while the blank control contained medium without cells. Sample evaluation was repeated four times. Briefly, the plate was incubated at $37^{\circ} \mathrm{C}$ in a $5 \% \mathrm{CO}_{2}$ incubator for $20 \mathrm{~h}$, and then $150 \mu \mathrm{l}$ 3-(4,5-dimethylthiazol-2-yl)-2,5-diphenyltetrazolium bromide (MTT) solution $(0.5 \mathrm{mg} / \mathrm{ml}$ in serum-free medium) was added to each well. After incubation at $37^{\circ} \mathrm{C}$ for $4 \mathrm{~h}$, the MTT solution was discarded, and $100 \mu \mathrm{l}$ dimethyl sulfoxide was added to each well. The plate was placed on a horizontal agitator for $10 \mathrm{~min}$, and the absorbance was determined at OD $570 \mathrm{~nm}$ using a microplate reader. The mean value of the four repeated tests was used, and the killing effect was calculated using the following equation:

$$
\text { Killing effect }=\left(1-\frac{\text { ODexp-ODctrE }}{\text { ODctrT }}\right) \times 100 \% \text {, }
$$

where ODexp is the absorbance value of the experimental wells, ODctrE represents the signal from the control wells of E cells and ODctrT represents the signal from the control wells of $\mathrm{T}$ cells.

Tumor formation in BALB/c nude mice. The LM8 murine osteosarcoma cells, which were stably transfected with the pGFP-V-RS, pGFP-V-RS-shRNA or pGFP-V-RS-shRNA-HSP70 vectors, were harvested in the logarithmic growth phase. The cells were centrifuged in a $10-\mathrm{ml}$ tube at $90 \times \mathrm{g}$ for $10 \mathrm{~min}$, and the supernatants were removed. Then, the cells were resuspended in serum-free DMEM $\left(5 \times 10^{7}\right.$ cells $\left./ \mathrm{ml}\right)$. A total of $18 \mathrm{BALB} / \mathrm{c}$ mice (4-6 week-old, males and females) were divided into three groups ( $n=6 /$ group). The cell suspension $(0.1 \mathrm{ml})$ was injected subcutaneously to the right sides of the back of the mice in the three groups, and tumor formation was observed every other day, with the longest (D) and shortest (d) diameter of the tumor being measured. The tumor volume was calculated using the following equation: Volume $=\mathrm{Dxd}^{2} / 2$.

After 40 days, the tumors were removed, weighed and fixed in $10 \%$ neutral formalin, followed by hematoxylin and eosin (H\&E) staining. 
A

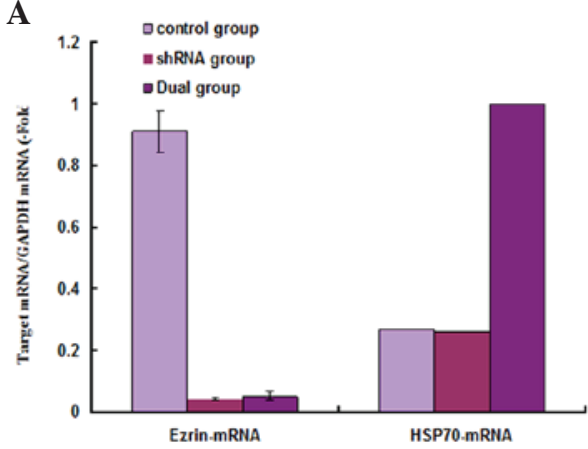

Ezrin-mRNA

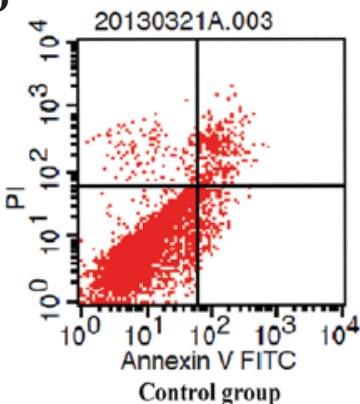

B

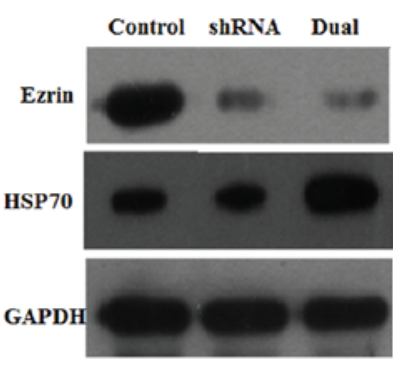

C

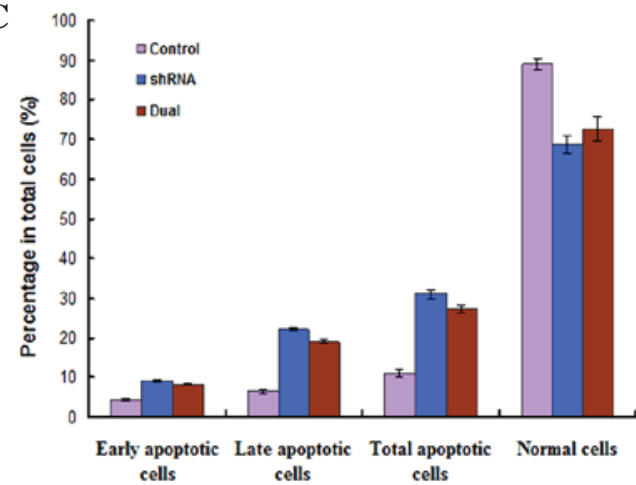

$\mathbf{E}$

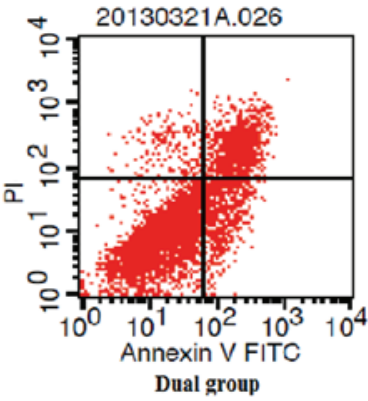

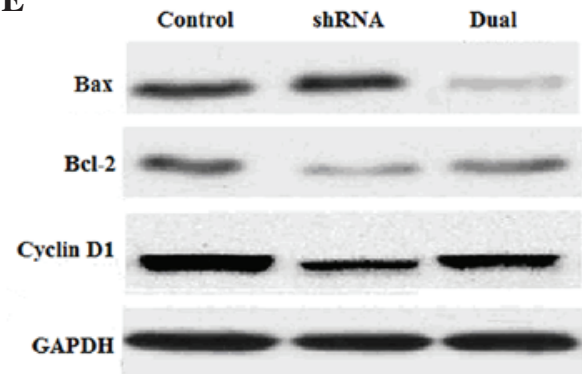

Figure 1. mRNA and protein expression of ezrin and HSP70 in transfected LM8 cells were detected by (A) reverse transcription-quantitative polymerase chain reaction and (B) western blotting, respectively. (C) Cellular apoptosis was analyzed by detecting the number of early and late apoptotic cells, as well as normal cells, in the NC, ezrin-shRNA and ezrin-shRNA/HSP70 groups. (D) Apoptosis analysis of stably transfected LM8 cells was performed by flow cytometry. (E) Apoptosis-associated proteins were detected by western blotting. NC, negative control; mRNA, messenger RNA; GAPDH, glyceraldehyde 3-phosphate dehydrogenase; shRNA, small hairpin RNA; HSP70, heat shock protein 70; Bcl-2, B-cell lymphoma-2; Bax, Bcl-2 associated X protein; PI, propidium iodide; FITC, fluorescein isothiocyanate.

Analysis of the splenic $T$ cell population in tumor-bearing mice. Blood samples from tumor-bearing mice were obtained from the orbital sinus. Tumor-bearing mice were sacrificed by cervical dislocation, and their spleens were removed with tweezers, placed on a steel mesh, cut into small pieces with scissors and ground with a mortar using 2-3 ml 1X PBS. The liquid was passed through the mesh into a culture dish and transferred to a 15-ml tube. After natural sedimentation for 3-5 min, the supernatant was transferred to a new 15-ml tube, and the sediment was discarded. Upon centrifugation at $200 \mathrm{x} \mathrm{g}$ for $6 \mathrm{~min}$, the supernatants were removed, and $1 \mathrm{ml}$ double distilled $\mathrm{H}_{2} \mathrm{O}$ was added to the sediment and mixed. After $30 \mathrm{sec}, 1 \mathrm{ml} 2 \mathrm{X}$ PBS was added, and the tube was centrifuged at $200 \mathrm{x} \mathrm{g}$ for $6 \mathrm{~min}$. The supernatant was removed, and the sediment was resuspended in $1.5 \mathrm{ml}$ PBS and then divided into $250 \mu \mathrm{l}$ aliquots in 6 centrifuge tubes $\left(\sim 1 \times 10^{5}\right.$ cells/tube). After centrifugation at $200 \mathrm{x} \mathrm{g}$ for $6 \mathrm{~min}$, the supernatants were removed, and $100 \mu \mathrm{l}$ of the corresponding fluorescent-labeled antibody was added to each tube. For the blank control, $100 \mu 1$ 1X PBS was added instead. Upon mixing, the cells were placed on ice away from light for $30 \mathrm{~min}$ and centrifuged at 2,000 rpm for $6 \mathrm{~min}$. The supernatants were removed, and $300 \mu 1 \mathrm{XX}$ PBS was added to each tube. Then, the cells were analyzed by flow cytometry.

Enzyme-linked immunosorbent assay (ELISA) analysis of IL-4 and interferon (IFN)- $\gamma$. The serum samples obtained from tumor-bearing mice in the control, shRNA and the dual groups were analyzed using an IL-4 or IFN- $\gamma$ ELISA kit (Thermo
Fisher Scientific, Inc.) according to the manufacturer's protocol. The experiment was repeated three times.

Ethics statement. The present study was undertaken according to the protocol approved by the constituted Ethics Committee of Xiamen University (Xiamen, China), which conforms to the principles of the Declaration of Helsinki.

Statistical analysis. Statistical analysis was performed using SPSS 17.0 software (SPSS, Inc., Chicago, IL, USA). Data were expressed as the mean \pm standard deviation. Multiple comparisons were evaluated by analysis of variance. For normally distributed data, the $t$ test was used for comparisons between groups; for non-normally distributed data, the Dunnett's $t$ test was used for comparisons between groups. $\mathrm{P}<0.05$ was considered to indicate a statistically significant difference.

\section{Results}

Expression of ezrin and HSP70 in transfected LM8 cells. In the shRNA and dual groups, the ezrin mRNA level was significantly lower than that in the control group $(\mathrm{P}<0.01)$. The RNA expression of ezrin was knocked down by $>99 \%$. Western blot analysis also revealed that ezrin protein expression was markedly decreased by ezrin-shRNA (Fig. 1A). The HSP70 mRNA level was significantly increased in the ezrin-shRNA/HSP70 group compared with the ezrin-shRNA and control groups $(\mathrm{P}=0.005)$. HSP70 protein overexpression was also confirmed by western blot analysis (Fig. 1B). 
Ezrin-shRNA/HSP70 transfection promotes the apoptosis of LM8 cells. As indicated in Fig. 1C and D, the apoptosis rate of LM8 cells was significantly increased in the shRNA group $(31.56 \pm 1.10 \%)$ compared with the control group $(11.01 \pm 0.80 \%)$ $(\mathrm{P}=0.023)$, particularly the rate of late apoptotic cells. When simultaneously overexpressing HSP70, the apoptosis rate of LM8 cells was slightly decreased compared with that of the shRNA group; however, the apoptosis rate $(27.31 \pm 0.95 \%)$ was still significantly higher than that in the control group $(11.01 \pm 0.80 \%)(\mathrm{P}=0.002)$.

In addition, western blot analysis demonstrated that ezrin-shRNA transfection promoted the expression of pro-apoptotic Bax, whereas it suppressed the expression of anti-apoptotic Bcl-2 and cyclin D1. The ezrin-shRNA/HSP70 group had overall weaker but similar effects regarding the expression of these proteins compared with the shRNA group. There were obvious differences compared with the control group (Fig. 1E).

Thus, stable transfection of ezrin-shRNA/HSP70 promoted the apoptosis of LM8 osteosarcoma cells.

Ezrin-shRNA/HSP70 transfection suppresses cell proliferation of LM8 cells. As represented in Fig. 2, the proliferation of LM8 cells in the three groups was analyzed by MTT assay. The absorbance value at day 0 was set to be $100 \%$. When compared with the control group, the proliferation rate in the shRNA group was significantly decreased from day 3 , and reached a peak at day $7(350.28 \pm 3.56$ vs. $190.76 \pm 4.71 \%)$ $(\mathrm{P}=0.001)$. The simultaneous reduction in ezrin expression and overexpression of HSP70 slightly recovered the cell proliferation decreased by ezrin knock-down. However, the proliferation rate was still significantly decreased at day 7 $(350.28 \pm 3.56$ vs. $280.61 \pm 3.23 \%)(\mathrm{P}=0.003)$. Thus, stable transfection of ezrin-shRNA/HSP70 suppressed the proliferation of LM8 osteosarcoma cells.

Cytotoxic effects of HSP70-induced CTL on LM8 cells. As indicated in Fig. 3, HSP70-induced CTL had a greater cytotoxic effect on LM8 cells, with a killing effect as high as $55.56 \pm 2.10 \%$, in the dual group. There were significant differences between the two experimental groups (shRNA and dual groups) and the control group $(\mathrm{P}=0.001)$. When comparing the CTL activity in different E:T ratio groups, HSP70-induced CTL had the highest killing effect on LM8 cells in the 100:1 (E:T) group.

Ezrin-shRNA/HSP70 transfection suppresses tumor formation in mice. Upon injection of stably transfected LM8 cells into nude mice, the tumor size and growth rate were analyzed. It was observed that the tumor growth was suppressed in the dual group 2-3 weeks after injection. There were significant differences in tumor growth rate compared with the shRNA and control groups $(\mathrm{P}=0.004)$ (Fig. 4A). After 6 weeks, the mice were sacrificed; the tumors were removed and weighed. The tumor weights in the dual group were significantly lower than those in the shRNA and control groups $(\mathrm{P}=0.029)$ (Fig. 4B and C).

Furthermore, histological examination of tumor sections by $\mathrm{H} \& \mathrm{E}$ staining revealed stroma-rich tumors with significantly smaller size and suppressed growth in the ezrin-shRNA/HSP70 group, compared with the other two groups (Fig. 4D). Thus,
Table I. Analysis of the T cell population of the tumorbearing mice.

\begin{tabular}{lccc}
\hline Group & $\begin{array}{c}\mathrm{CD}^{+} / \mathrm{CD}^{+} \\
(\%)\end{array}$ & $\begin{array}{c}\mathrm{CD}^{+} / \mathrm{CD}^{+} \\
(\%)\end{array}$ & $\begin{array}{c}\mathrm{CD}^{+} / \mathrm{CD}^{+} \\
\text {(ratio) }\end{array}$ \\
\hline Control group & $23.56 \pm 2.30$ & $18.29 \pm 2.10$ & $1.279 \pm 0.036$ \\
shRNA group & $22.49 \pm 1.60$ & $19.14 \pm 2.30$ & $1.182 \pm 0.052$ \\
Dual group & $17.01 \pm 1.40$ & $27.91 \pm 2.60$ & $0.623 \pm 0.042^{\mathrm{a}}$ \\
\hline
\end{tabular}

${ }^{a} \mathrm{P}<0.01$ vs. shRNA group or vs. control group. Data are presented as the mean \pm standard deviation. CD, cluster of differentiation; shRNA, small hairpin RNA.

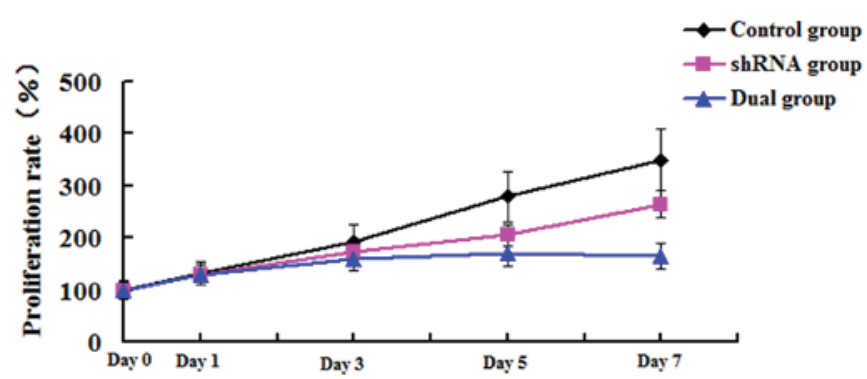

Figure 2. MTT analysis of stably transfected LM8 cells. Cell proliferation was analyzed for 7 days by MTT assay in the negative control, ezrin-shRNA and ezrin-shRNA/HSP70 groups. The absorbance of cells at day 0 was considered as $100 \%$. shRNA, small hairpin RNA; MTT, 3-(4,5-dimethylthiazol-2-yl)-2,5-diphenyltetrazolium bromide; HSP70, heat shock protein 70 .

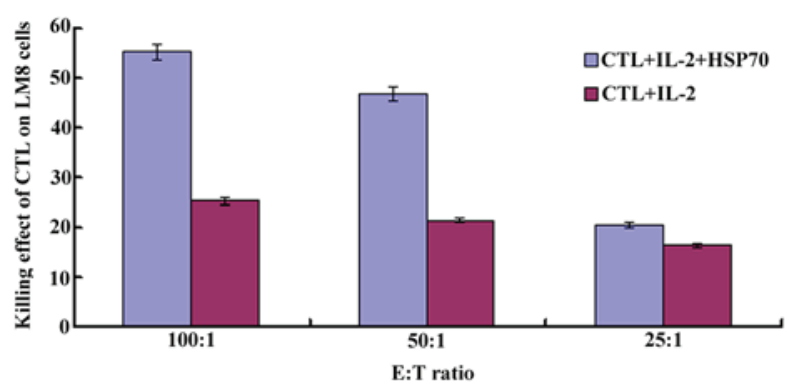

Figure 3. Cytotoxic effect of HSP70-induced CTL activity on LM8 cells. The E:T ratios of 100:1, 50:1 and 25:1 were used, and the killing effect of HSP70-induced CTL activity on LM8 osteosarcoma cells was analyzed by 3-(4,5-dimethylthiazol-2-yl)-2,5-diphenyltetrazolium bromide assay. The data is presented as the mean \pm standard deviation. CTL, cytotoxic T lymphocyte; HSP70, heat shock protein 70; IL-2, interleukin-2; E, effector cells; T, target cells.

stable transfection of ezrin-shRNA/HSP70 in LM8 osteosarcoma cells suppressed tumor formation in nude mice.

Transfection of ezrin-shRNA/HSP70 increases cluster of differentiation $(C D) 8^{+} T$ cells in tumor-bearing mice. As indicated in Table I and Fig. 5, the percentage of $\mathrm{CD}^{+} \mathrm{T}$ lymphocytes was increased, whereas the percentage of $\mathrm{CD}^{+} \mathrm{T}$ lymphocytes was decreased, in the ezrin-shRNA/HSP70 group, and the ratio of $\mathrm{CD}^{+} / \mathrm{CD}^{+} \mathrm{T}$ lymphocytes was significantly smaller than that in the shRNA and control groups $(\mathrm{P}=0.006)$. There were no significant differences between the shRNA group and the control group $(\mathrm{P}=0.102)$. 
A

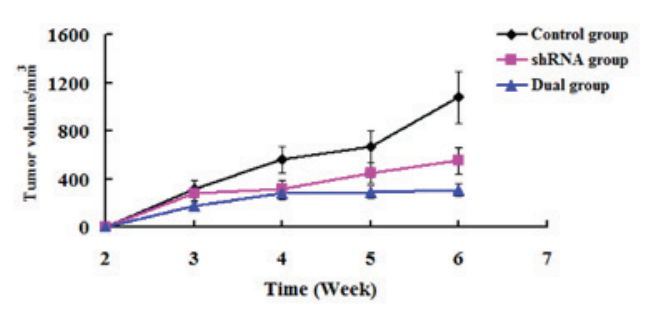

D
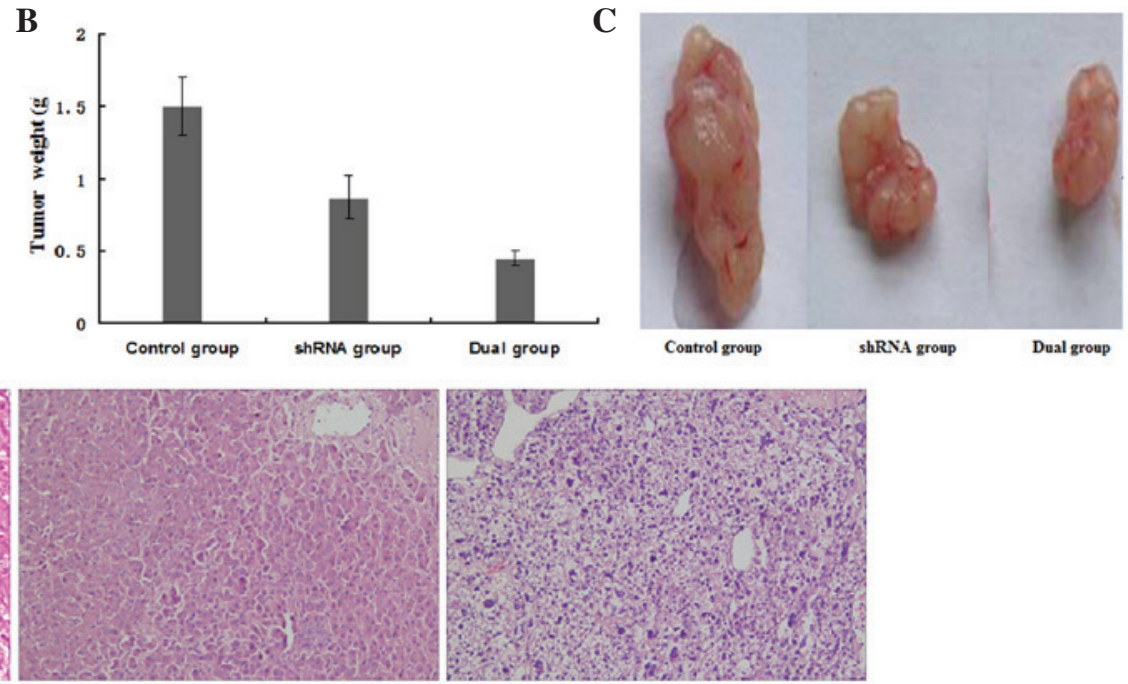

shRNA group

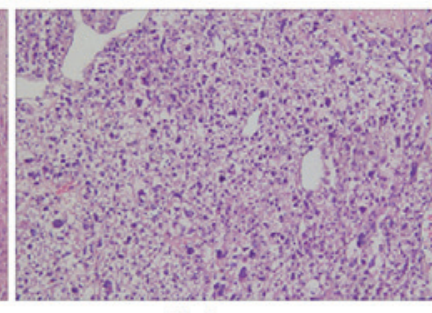

Dual group

Figure 4. Tumor formation and characterization upon injected LM8 cells were transfected with different vectors. (A) Tumor growth in mice injected with stably transfected LM8 cells. (B) Tumor weight in mice 6 weeks after injection. (C) Image of tumors in mice (magnification, x100). (D) Histopathological changes of tumors from tumor-bearing mice (magnification, x100). Tumors were obtained from mice in the negative control, ezrin-shRNA and ezrin-shRNA/HSP70 (dual) groups 6 weeks after injection, and subjected to histopathological analysis using hematoxylin and eosin staining. shRNA, small hairpin RNA; HSP70, heat shock protein 70 .

A
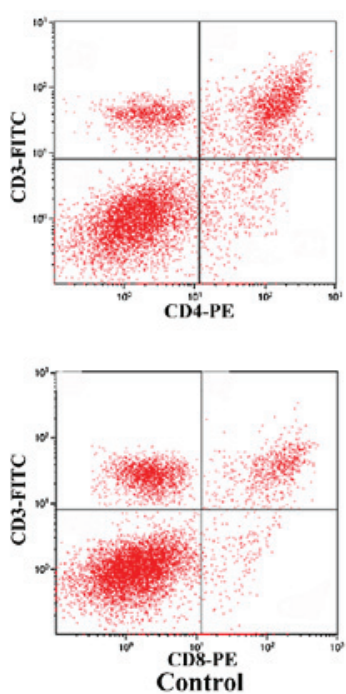
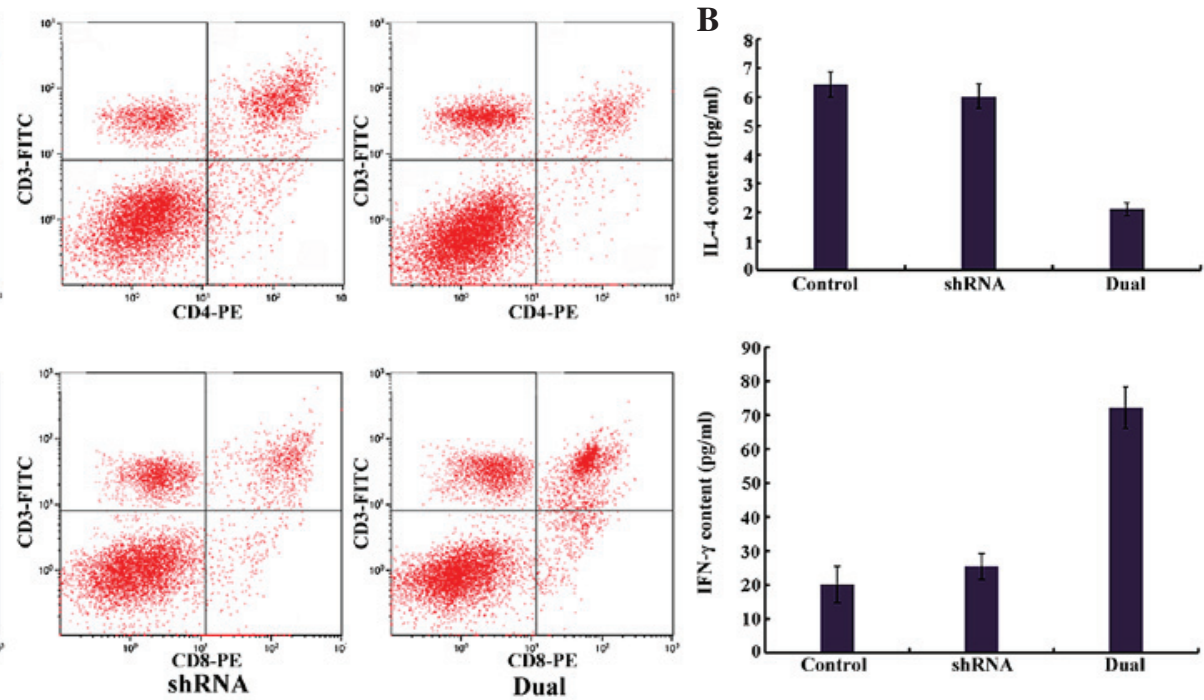

Figure 5. Immunological analysis of tumor-bearing mice. (A) Analysis of the splenic T cell population from tumor-bearing mice. At 40 days after injection, spleens were removed from the mice, and the splenic T cell population was analyzed by flow cytometry. (B) Analysis of serum IL-4 and IFN- $\gamma$ levels in tumor-bearing mice. At 40 days after injection, the serum samples were collected from the tumor-bearing mice, and the levels of IL- 4 and IFN- $\gamma$ were determined by enzyme-linked immunosorbent assay. CD, cluster of differentiation; IL, interleukin; IFN, interferon; shRNA, small hairpin RNA; PE, phycoerythrin; FITC, fluorescein isothiocyanate.

Transfection of ezrin-shRNA/HSP70 promotes immunological killing of tumors. As represented in Fig. 5B, in the dual group, the serum IL-4 level was significantly decreased, and the serum IFN- $\gamma$ level was significantly increased, compared with the ezrin-shRNA and negative control (NC) groups $(\mathrm{P}<0.01)$, whereas there were no significant differences in IL- 4 or IFN- $\gamma$ levels between the ezrin-shRNA group and the NC group ( $\mathrm{P}>0.05)$.

\section{Discussion}

It has been reported that downregulation of ezrin expression by small interfering (si)RNA (12) or alteration of its phosphorylation (10) resulted in the apoptosis of osteosarcoma cells and a reduced survival rate of tumor cells. However, the siRNA technique has certain disadvantages, including low effectiveness and instability, whereas the shRNA technique can establish stable, long-term gene silencing cell lines with high success rates (24). In the present study, shRNA eukaryotic expression vectors were used to continuously downregulate ezrin expression. After 1 month of selection, the LM8 cell lines that silenced ezrin gene expression were established and confirmed by fluorescence microscopy. The efficiency of gene silencing ezrin was $>99 \%$, indicating that the shRNA expression vector is an efficient method to downregulate ezrin 
gene expression. When comparing cells with or without gene silencing, it was noticed that the apoptosis rate was significantly increased, while the cell proliferation rate was significantly reduced, upon reducing ezrin expression, and thus, the tumor cell growth was effectively inhibited.

However, even though the ezrin gene was almost completely silenced, the growth rate of LM8 cells was inhibited only by $45.7 \%$. In addition, the transgenic technology is unable to transfect all tumor cells. Therefore, it is difficult to completely remove all tumor cells, which is an important reason for incomplete treatment, and ultimately leads to tumor recurrence and metastasis (25). Wang et al reported that immunization of mice with HSP70 extracted from tumor tissues could induce specific CTL activity and tumor killing effects (26). That study was performed in a mouse model of colon cancer. In the current study, the pGFP-V-RS vector, which contains a cytomegalovirus promoter and a U6 promoter, was constructed to simultaneously silence ezrin gene expression while producing HSP70 overexpression, and to establish stably transfected cell lines. HSP70 overexpression partially recovered the promoted cellular apoptosis and proliferation suppression by knocking down ezrin. However, when compared with the normal control, HSP70 overexpression significantly inhibited the tumor cell growth and induced the apoptosis of tumor cells. At the same time, the overexpressed HSP70 protein could be released by the apoptotic tumor cells and act as a danger signal, further inducing the specific immune response against osteosarcoma cells, and thus, was able to remove tumor cells to a greater extent.

In the present study, tumor-bearing mice models were established by injecting the stably transfected murine LM8 osteosarcoma cells with pGFP-V-RS, pGFP-V-RS-shRNA and pGFP-V-RS-shRNA-HSP70. The results demonstrated that mice in the ezrin-shRNA/HSP70 group experienced higher anti-tumor effects than those in the control group, since histopathological examination of their tumor sections revealed more necrotic tissues and smaller volumes of tumor cells compared with the control mice. Flow cytometric analysis demonstrated that, in the ezrin-shRNA/HSP70 group, the percentage of $\mathrm{CD}^{+} \mathrm{T}$ lymphocytes in spleen was higher, and the ratio of $\mathrm{CD}^{+} / \mathrm{CD}^{+} \mathrm{T}$ lymphocytes was lower, compared with the control group; this suggests that HSP70 is important in tumor-antigen presentation and CD8 ${ }^{+}$ $\mathrm{T}$ lymphocyte activation. Serum levels of IL- 4 and IFN- $\gamma$ in tumor-bearing mice were measured by ELISA,which revealed that, in the ezrin-shRNA/HSP70 group, the IFN- $\gamma$ level was significantly increased, while the IL-4 level was significantly decreased. This suggests that, once the antigen was presented, $\mathrm{T}$ cells and NK cells were activated, thus secreting IFN- $\gamma$ and consequently activating macrophages, dendritic cells and NK cells, and triggering cell-mediated immunity to inhibit tumor growth. Whereas the humoral immunity was suppressed, the IL-4 level was decreased. These results were consistent with our recent findings in human osteosarcoma cells (23).

Overall, it was speculated that, in the tumor tissues of mice in the ezrin-shRNA/HSP70 group, ezrin-shRNA induced the release of overexpressed HSP70 protein from tumor cells; then, the HSP70-bound tumor-derived mutated antigens and the HSP70-peptide complexes were recognized by the pattern recognition receptors on the surface of APCs and subsequently phagocytosed by APCs. Thus, the immune tolerance was broken, and the tumor killing effects of $\mathrm{T}$ lymphocytes and NK cells were induced.

In summary, the results of the present study suggested that stable transfection of the specific pGFP-V-RS-shRNA-HSP70 vector induced apoptosis and reduced the proliferation rate of osteosarcoma cells, and in combination with HSP70-induced cellular immune response, it also induced the amplification of tumor killing effects. The present study has assessed the effects of the ezrin-shRNA/HSP70 transfection method on tumor treatment in vitro and in vivo, and provides the theoretical and experimental basis for clinical application of gene therapy for osteosarcoma.

\section{References}

1. Janeway KA, Barkauskas DA, Krailo MD, Meyers PA, Schwartz CL, Ebb DH, Seibel NL, Grier HE, Gorlick R and Marina N: Outcome for adolescent and young adult patients with osteosarcoma: A report from the children's oncology group: Cancer 118: 4597-4605, 2012.

2. Bacci G,Ferrari S, Longhi A, Donati D, Manfrini M, Giacomini S, Briccoli A, Forni C and Galletti S: Nonmetastatic osteosarcoma of the extremity with pathologic fracture at presentation: Local and systemic control by amputation or limb salvage after preoperative chemotherapy. Acta Orthop Scand 74: 449-454, 2003.

3. Gobin B, Moriceau G, Ory B, Charrier C, Brion R, Blanchard F, Redini $\mathrm{F}$ and Heymann D: Imatinib mesylate exerts anti-proliferative effects on osteosarcoma cells and inhibits the tumour growth in immunocompetent murine models. PLoS One 9: e90795, 2014.

4. Arias JL: Drug targeting strategies in cancer treatment: An overview. Mini Rev Med Chem 11, 1-17, 2011.

5. De Santi C, Pietrabissa A, Mosca F and Pacifici GM: Methylation of quercetin and fisetin, flavonoids widely distributed in edible vegetables, fruits and wine, by human liver. Int J Clin Pharmacol Ther 40: 207-212, 2002.

6. Khanna C, Wan X, Bose S, Cassaday R, Olomu O, Mendoza A, Yeung C, Gorlick R, Hewitt SM and Helman LJ: The membrane-cytoskeleton linker ezrin is necessary for osteosarcoma metastasis. Nat Med 10: 182-186, 2004.

7. Ren L, Hong SH, Cassavaugh J, Osborne T, Chou AJ, Kim SY, Gorlick R, Hewitt SM and Khanna C: The actin-cytoskeleton linker protein ezrin is regulated during osteosarcoma metastasis by PKC. Oncogene 28: 792-802, 2009.

8. Zhang Y, Zhang L, Zhang G, Li S, Duan J, Cheng J, Ding G, Zhou C, Zhang J, Luo P, et al: Osteosarcoma metastasis: Prospective role of ezrin. Tumour Biol 35, 5055-5059, 2014.

9. Lin LJ and Chen LT: Association between ezrin protein expression and the prognosis of colorectal adenocarcinoma. Mol Med Rep 8: 61-66, 2013.

10. Li L, Wang YY, Zhao ZS and Ma J: Ezrin is associated with gastric cancer progression and prognosis. Pathol Oncol Res 17: 909-915, 2011.

11. Lo Vasco VR, Leopizzi M, Puggioni C and Della Rocca C: Ezrin silencing remodulates the expression of Phosphoinositide-specific Phospholipase $\mathrm{C}$ enzymes in human osteosarcoma cell lines. J Cell Commun Signal 8: 219-229, 2014.

12. Shang X, Wang Y, Zhao Q, Wu K, Li X, Ji X, He R and Zhang W: siRNAs target sites selection of ezrin and the influence of RNA interference on ezrin expression and biological characters of osteosarcoma cells. Mol Cell Biochem 364: 363-371, 2012.

13. Yang T, Zhang H, Cai SY, Shen YN, Yuan SX, Yang GS, Wu MC, Lu JH and Shen F: Elevated SHOX2 expression is associated with tumor recurrence of hepatocellular carcinoma. Ann Surg Oncol 20 (Suppl 3): S644-S649, 2013.

14. Murugesan SR, Akiyama M, Einfeld DA, Wickham TJ and King CR: Experimental treatment of ovarian cancers by adenovirus vectors combining receptor targeting and selective expression of tumor necrosis factor. Int J Oncol 31: 813-822, 2007.

15. Cai G, Zhu X, Xu Y, Du X, Zhang Z, Zhang Y, Chen T, Zhou X, Guan Z and Cai S: Case report of extrarenal rhabdoid tumor of pelvic retroperitoneum molecular profile of angiogenesis and its implication in new treatment strategy. Cancer Biol Ther 8: 417-421, 2009. 
16. Goloudina AR, Demidov ON and Garrido C: Inhibition of HSP70: A challenging anti-cancer strategy. Cancer Lett 325: 117-124, 2012.

17. Sliutz G, Karlseder J, Tempfer C, Orel L, Holzer G and Simon MM: Drug resistance against gemcitabine and topotecan mediated by constitutive hsp70 overexpression in vitro: Implication of quercetin as sensitiser in chemotherapy. Br J Cancer 74: 172-177, 1996.

18. Sashchenko LP, Dukhanina EA, Shatalov YV, Yashin DV, Lukyanova TI, Kabanova OD, Romanova EA, Khaidukov SV, Galkin AV, Gnuchev NV and Georgiev GP: Cytotoxic T lymphocytes carrying a pattern recognition protein Tag7 can detect evasive, HLA-negative but Hsp70-exposing tumor cells, thereby ensuring FasL/Fas-mediated contact killing. Blood 110 1997-2004, 2007.

19. Stangl S, Gross C, Pockley AG, Asea AA and Multhoff G: Influence of Hsp70 and HLA-E on the killing of leukemic blasts by cytokine/Hsp70 peptide-activated human natural killer (NK) cells. Cell Stress Chaperones 13, 221-230, 2008.

20. Zitzler S, Hellwig A, Hartl FU, Wieland F and Diestelkötter-Bachert P: Distinct binding sites for the ATPase and substrate-binding domain of human Hsp70 on the cell surface of antigen presenting cells. Mol Immunol 45: 3974-3983, 2008.
21. Nishikawa M, Otsuki T, Ota A, Guan X, Takemoto S, Takahashi Y and Takakura Y: Induction of tumor-specific immune response by gene transfer of Hsp70-cell-penetrating peptide fusion protein to tumors in mice. Mol Ther 18: 421-428, 2010.

22. Yamaoka A, Guan X, Takemoto S, Nishikawa M and Takakura Y Development of a novel Hsp70-based DNA vaccine as a multifunctional antigen delivery system. J Control Release 142: 411-415, 2010.

23. Yao Q, Zhao HY and Xie BZ: effects of ezrin and heat shock protein 70 on apoptosis and proliferation of human osteosarcoma cells. Orthop Surg 7: 273-280, 2015.

24. Rácz Z and Hamar P: SiRNA technology, the gene therapy of the future? Orv Hetil 149: 153-159, 2008 (In Hungarian).

25. Wirtzfeld LA, Wu G, Bygrave M, Yamasaki Y, Sakai H, Moussa M, Izawa JI, Downey DB, Greenberg NM, Fenster A, et al: A new three-dimensional ultrasound microimaging technology for preclinical studies using a transgenic prostate cancer mouse model. Cancer Res 65: 6337-6345, 2005.

26. Wang ZH, Ye Q, Hu ZQ, Ye ZQ, Yu X and Shen GX: In vitro anti-tumor effect of CTL induced by HSP70-Id complex-modified dendritic cells. Zhonghua Zhong Liu Za Zhi 28: 481-485, 2006 (In Chinese) 